\title{
Influence mechanism of excipients on drug crystallization: experimental investigation and model analysis and prediction
}

\author{
Qiao Chen ${ }^{1}$, Jingyun Weng ${ }^{1}$, Gabriele Sadowski ${ }^{2}$, and Yuanhui $\mathrm{Ji}^{1}$ \\ ${ }^{1}$ Southeast University \\ ${ }^{2}$ TU Dortmund
}

January 11, 2022

\begin{abstract}
The influence of temperature, stirring speed, and excipients on crystal growth kinetics of mesalazine and allopurinol was investigated through experiment and chemical potential gradient model. The results indicated that the Diffusion-Surface Reaction model (DSR $(1,2)$ ) showed good performance in modeling API crystal growth kinetics within the ARDs of $4 \%$. Excipients played a crucial role in inhibiting crystal growth in all the systems. It can not only improve the API solubility, but also reduce the crystal growth rate. By comparing diffusion rate and surface-reaction rate constant within the DSR $(1,2)$ model, it was found that the controlling step of mesalazine crystallization was surface-reaction. Allopurinol crystallization was dominated by both surface-reaction and diffusion. Meanwhile, the crystal growth kinetics of mesalazine and allopurinol were predicted successfully with the ARDs of $2.53 \%$ and $4.78 \%$. This work provided a mechanistic understanding of polymer influence on the inhibition of API crystal growth.
\end{abstract}

\section{Hosted file}

Manuscrip.docx available at https://authorea.com/users/454892/articles/552365-influencemechanism-of-excipients-on-drug-crystallization-experimental-investigation-and-modelanalysis-and-prediction 